\title{
Coupled Electron- and Phase-Transfer Reactions at a Three-Phase Interface
}

\author{
Connor K. Terry Weatherly, Hang Ren, Martin A. Edwards, Li Wang, and Henry S. White* \\ Department of Chemistry, University of Utah, Salt Lake City, UT 84112, United States \\ *Corresponding author: white@chem.utah.edu
}

Below is the automatically generated Comsol "model report", which describes in detail the boundary conditions, geometry, mesh, solver configuration, etc., used in the finite element simulations of the 3-phase interface reported in this work and described in Supporting Information, section 6. 


\section{Contents}

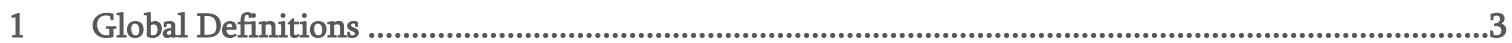

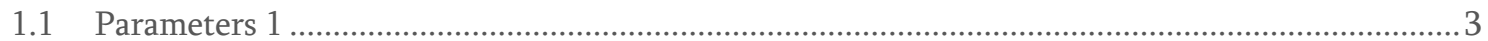

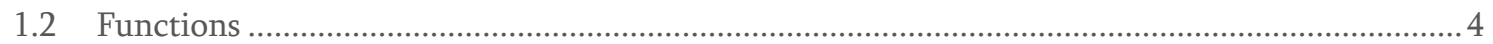

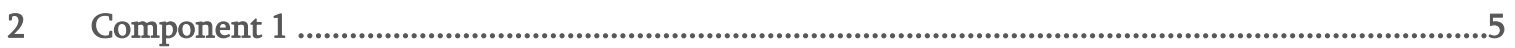

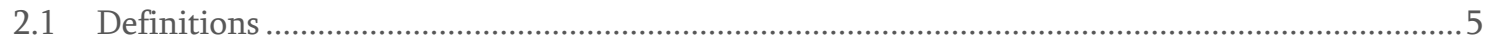

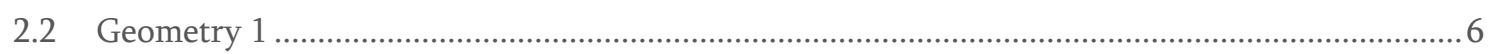

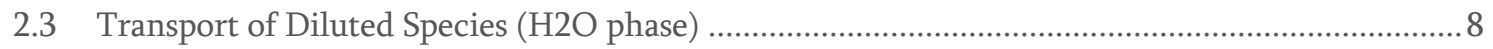

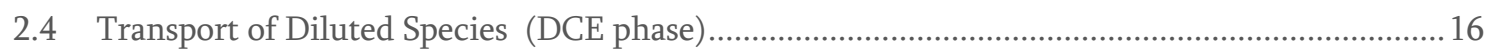

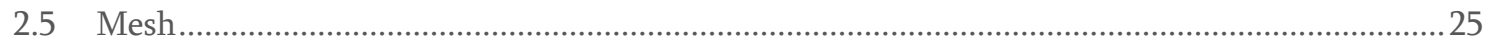

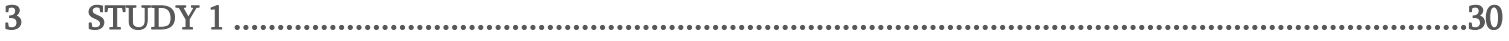

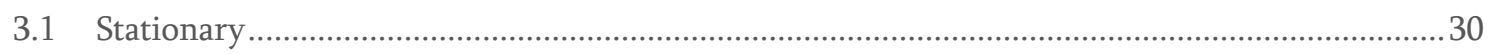

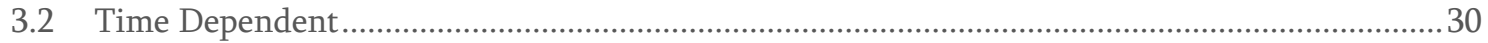

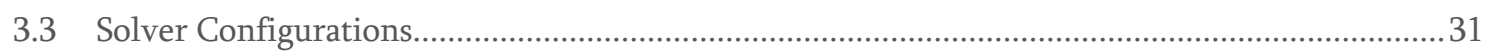




\section{Global Definitions}

Date Jul 9, 2018 1:29:50 PM

\section{GLOBAL SETTINGS}

\begin{tabular}{|l|l|}
\hline Name & Basic LLS 3phase Model Report.mph \\
\hline Path & D:\Google Drive\Hang Ren\Interface_Wire_ET\Basic_LLS_3phase_Model_Report.mph \\
\hline Version & COMSOL Multiphysics 5.3a (Build: 275)
\end{tabular}

\section{USED PRODUCTS}

COMSOL Multiphysics

Chemical Reaction Engineering Module

\subsection{PARAMETERS 1}

\section{PARAMETERS}

\begin{tabular}{|c|c|c|c|}
\hline Name & Expression & Value & Description \\
\hline alpha & 0.5 & 0.5 & transfer coefficient \\
\hline C_Fc_org & $5[\mathrm{mmol} / \mathrm{L}]$ & $5 \mathrm{~mol} / \mathrm{m}^{3}$ & bulk concentration of Fc in DCE \\
\hline D_Fc_aq & $0.50 \mathrm{e}-5\left[\mathrm{~cm}^{\wedge} 2 / \mathrm{s}\right]$ & $5 \mathrm{E}-10 \mathrm{~m}^{2} / \mathrm{s}$ & Diffusion coefficient of $\mathrm{Fc}_{\mathrm{c}}$ in $\mathrm{H}_{2} \mathrm{O}$ \\
\hline D_Fc_org & $0.95 \mathrm{e}-5\left[\mathrm{~cm}^{\wedge} 2 / \mathrm{s}\right]$ & $1 \mathrm{E}-9 \mathrm{~m}^{2} / \mathrm{s}$ & Diffusion coefficient of Fc in DCE \\
\hline D_Fc_plus_aq & $1.0 \mathrm{e}-5\left[\mathrm{~cm}^{\wedge} 2 / \mathrm{s}\right]$ & $1 \mathrm{E}-9 \mathrm{~m}^{2} / \mathrm{s}$ & Diffusion coefficient of $\mathrm{Fc}^{+}$in $\mathrm{H}_{2} \mathrm{O}$ \\
\hline D_Fc_plus_org & $2.0 \mathrm{e}-5\left[\mathrm{~cm}^{\wedge} 2 / \mathrm{s}\right]$ & $2 \mathrm{E}-9 \mathrm{~m}^{2} / \mathrm{s}$ & Diffusion coefficient of $\mathrm{Fc}^{+}$in DCE \\
\hline E0_aq & $0.32[\mathrm{~V}]$ & $0.32 \mathrm{~V}$ & $E^{0 \prime}$ of $\mathrm{Fc} / \mathrm{Fc}^{+}$in $\mathrm{H}_{2} \mathrm{O}(\mathrm{vs} \mathrm{Ag} / \mathrm{AgCl})$ \\
\hline E0_org & $0.55[\mathrm{~V}]$ & $0.55 \mathrm{~V}$ & $E^{0 \prime}$ of $\mathrm{Fc} / \mathrm{Fc}^{+}$in $\mathrm{DCE}(\mathrm{vs} \mathrm{Ag} / \mathrm{AgCl})$ \\
\hline $\mathrm{Ef}$ & $0.9[\mathrm{~V}]$ & $0.9 \mathrm{~V}$ & switching potential \\
\hline $\mathrm{Ei}$ & $0.1[\mathrm{~V}]$ & $0.1 \mathrm{~V}$ & initial potential \\
\hline$f$ & $\mathrm{~F} / \mathrm{R} / \mathrm{T}$ & $39.6081 / \mathrm{V}$ & \\
\hline $\mathrm{F}$ & $96485[\mathrm{C} / \mathrm{mol}]$ & $96485 \mathrm{C} / \mathrm{mol}$ & Faraday's constant \\
\hline $\mathrm{k} \_0$ & $0.5[\mathrm{~cm} / \mathrm{s}]$ & $0.005 \mathrm{~m} / \mathrm{s}$ & $\begin{array}{l}\text { heterogeneous rate constant of } \\
\text { electron transfer for Fc in DCE }\end{array}$ \\
\hline P_Fc_plus & 0.1 & 0.1 & $\begin{array}{l}\text { partition coefficient for } \mathrm{Fc}^{+} \\
\left(\mathrm{C}_{\mathrm{aq}} / \mathrm{Corg}\right)\end{array}$ \\
\hline P_Fc & $1 \mathrm{e} 6$ & $1 \mathrm{E} 6$ & partition coefficient for $\mathrm{Fc}_{\mathrm{c}}\left(\mathrm{Caq}_{\mathrm{a}} / \mathrm{Corg}\right)$ \\
\hline $\mathrm{kpt}$ & $10[\mathrm{~cm} / \mathrm{s}]$ & $0.1 \mathrm{~m} / \mathrm{s}$ & $\begin{array}{l}\text { heterogeneous rate constant of } \\
\text { phase transfer reactions }\end{array}$ \\
\hline $\mathrm{R}$ & $8.314[\mathrm{~J} / \mathrm{K} / \mathrm{mol}]$ & $8.314 \mathrm{~J} /(\mathrm{mol} \cdot \mathrm{K})$ & gas constant \\
\hline $\mathrm{T}$ & $293[\mathrm{~K}]$ & $293 \mathrm{~K}$ & thermodynamic temperature \\
\hline ts & $\operatorname{abs}((E f-E i) / v)$ & $8 \mathrm{~s}$ & Duration of 1 segment of CV. \\
\hline $\mathrm{v}$ & $0.1[\mathrm{~V} / \mathrm{s}]$ & $0.1 \mathrm{~V} / \mathrm{s}$ & scan rate \\
\hline
\end{tabular}




\begin{tabular}{|l|l|l|l|}
\hline Name & Expression & Value & Description \\
\hline WireRadius & $0.125[\mathrm{~mm}]$ & $1.25 \mathrm{E}-4 \mathrm{~m}$ & radius of the Pt wire electrode \\
\hline domain_size & $1250[\mathrm{um}]$ & $0.00125 \mathrm{~m}$ & \\
\hline wire_depth & $750[\mathrm{um}]$ & $7.5 \mathrm{E}-4 \mathrm{~m}$ & $\begin{array}{l}\text { length of wire dipped into the } \\
\text { organic phase }\end{array}$ \\
\hline $\mathrm{t}$ & $0[\mathrm{~s}]$ & $0 \mathrm{~s}$ & $\begin{array}{l}\text { tofor Stationary Study. } \\
\text { heterogeneous rate constant of } \\
\text { electron transfer for } \mathrm{Fc} \text { in } \mathrm{H}_{2} \mathrm{O}\end{array}$ \\
\hline $\mathrm{k} \_0 \_$aq & $0.5[\mathrm{~cm} / \mathrm{s}]$ & $0.005 \mathrm{~m} / \mathrm{s}$ & \\
\hline
\end{tabular}

\subsection{FUNCTIONS}

\subsubsection{Interpolation 2}

\begin{tabular}{|l|l|}
\hline Function names & Et \\
\hline Function type & Interpolation \\
\hline
\end{tabular}

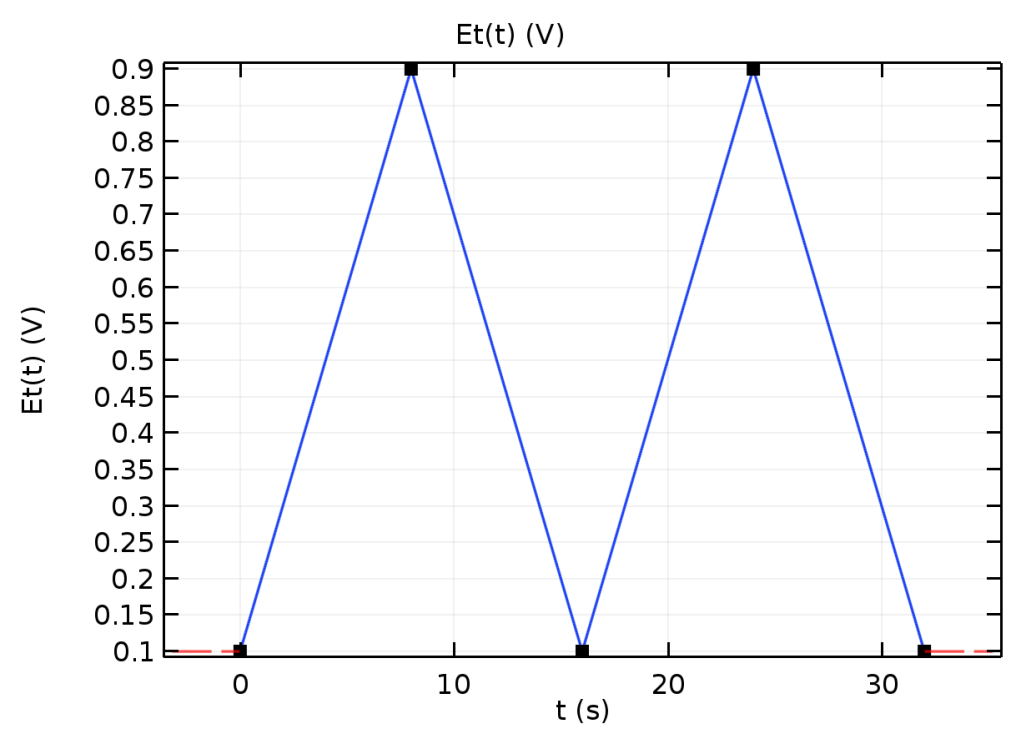

Interpolation 2

UNITS

\begin{tabular}{|l|l|}
\hline Description & Value \\
\hline Arguments & $\mathrm{s}$ \\
\hline Function & $\mathrm{V}$ \\
\hline
\end{tabular}




\section{Component 1}

SETTINGS

\begin{tabular}{|l|l} 
Description & Value \\
\hline Unit system & Same as global system
\end{tabular}

\subsection{DEFINITIONS}

\subsubsection{Variables}

water

SELECTION

\begin{tabular}{|l|l|}
\hline Geometric entity level & Domain \\
\hline Selection & Domain 2 \\
\hline
\end{tabular}

\begin{tabular}{|l|l|l|l|}
\hline Name & Expression & Unit & Description \\
\hline Fc_plus & Fc_plus_aq & $\mathrm{mol} / \mathrm{m}^{3}$ & Ferrocenium in $\mathrm{H}_{2} \mathrm{O}$ phase \\
\hline Fc & Fc_aq & $\mathrm{mol} / \mathrm{m}^{3}$ & Ferrocene in $\mathrm{H}_{2} \mathrm{O}$ phase \\
\hline
\end{tabular}

org

SELECTION

Geometric entity level Domain

Selection Domain 1

\begin{tabular}{|l|l|l|l|}
\hline Name & Expression & Unit & Description \\
\hline Fc_plus & Fc_plus_org & $\mathrm{mol} / \mathrm{m}^{3}$ & Ferrocenium in organic phase \\
\hline Fc & Fc_org & $\mathrm{mol} / \mathrm{m}^{3}$ & Ferrocene in organic phase \\
\hline
\end{tabular}

\subsubsection{Coordinate Systems}

Boundary System 1

\begin{tabular}{|l|l|}
\hline Coordinate system type & Boundary system \\
\hline Tag & sys1 \\
\hline
\end{tabular}

SETTINGS

\begin{tabular}{|l|l|l|} 
First & Second & Third \\
\hline t1 & to & n \\
\hline
\end{tabular}




\subsection{GEOMETRY 1}

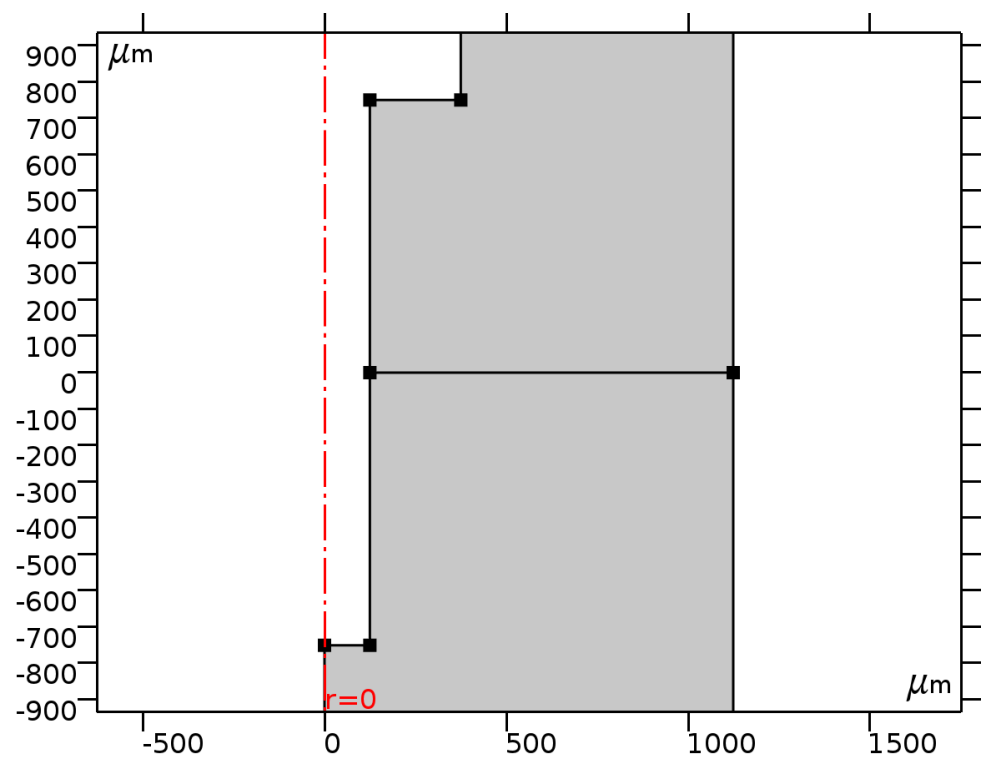

Geometry 1

UNITS

Length unit $\mu \mathrm{m}$

Angular unit deg

GEOMETRY STATISTICS

\begin{tabular}{l|l} 
Description & Value \\
\hline Space dimension & 2 \\
\hline Number of domains & 2 \\
\hline Number of boundaries & 11 \\
\hline Number of vertices & 10
\end{tabular}

\subsubsection{Rectangle 2 (r2)}

\section{POSITION}

\section{Description Value}

Position \{WireRadius, 0\}

SIZE

\begin{tabular}{|l|l|}
\hline Description & Value \\
\hline Width & domain_size ${ }^{*} 0.8$ \\
\hline Height & domain_size \\
\hline
\end{tabular}




\subsubsection{Rectangle 3 (r3)}

\section{POSITION}

\begin{tabular}{|l|l|}
\hline Description & Value \\
\hline Position & \{WireRadius, -domain_size \\
\hline
\end{tabular}

SIZE

\begin{tabular}{|l|l|}
\hline Description & Value \\
\hline Width & domain_size* 0.8 \\
\hline Height & domain_size \\
\hline
\end{tabular}

\subsubsection{Point 1 (pt1)}

\section{POINT}

\begin{tabular}{|l|l|}
\hline Description & Value \\
\hline Point coordinate & $\{$ WireRadius, 750 \\
\hline
\end{tabular}

\subsubsection{Point 2 (pt2)}

POINT

\begin{tabular}{|l|l|}
\hline Description & Value \\
\hline Point coordinate & $\{$ WireRadius, -750$\}$ \\
\hline
\end{tabular}

\subsubsection{Rectangle 4 (r4)}

$$
\text { POSITION }
$$

\begin{tabular}{|l|l|}
\hline Description & Value \\
\hline Position & $\{0$, -domain_size $\}$ \\
\hline
\end{tabular}

$$
\text { SIZE }
$$

\begin{tabular}{|l|l|}
\hline Description & Value \\
\hline Width & WireRadius \\
\hline Height & domain_size-wire_depth \\
\hline
\end{tabular}

\subsubsection{Rectangle 5 (r5)}

\section{POSITION}

\begin{tabular}{l|l} 
Description & Value \\
\hline Position & \{WireRadius, domain_size-(domain_size-wire_depth)
\end{tabular}

\begin{tabular}{|l|l|}
\hline Description & Value \\
\hline Width & WireRadius*2 \\
\hline
\end{tabular}




\begin{tabular}{|l|l|}
\hline Description & Value \\
\hline Height & domain_size-wire_depth \\
\hline
\end{tabular}

\subsection{TRANSPORT OF DILUTED SPECIES (H2O PHASE)}

\section{USED PRODUCTS}

COMSOL Multiphysics

Chemical Reaction Engineering Module

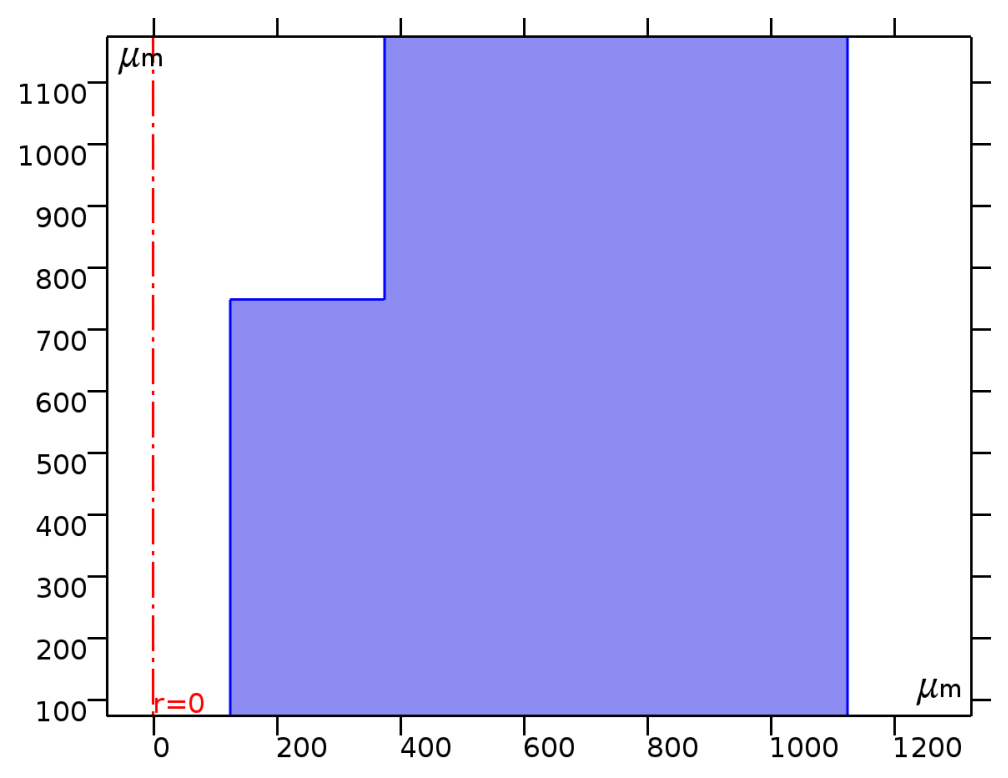

Transport of Diluted Species (H2O phase)

\section{SELECTION}

\begin{tabular}{|l|l|}
\hline Geometric entity level & Domain \\
\hline Selection & Domain 2 \\
\hline
\end{tabular}

EQUATIONS

$$
\begin{aligned}
& \frac{\partial c_{i}}{\partial \mathrm{t}}+\nabla \cdot\left(-D_{i} \nabla c_{i}\right)=R_{i} \\
& \mathbf{N}_{i}=-D_{i} \nabla c_{i}
\end{aligned}
$$

\subsubsection{Interface settings}

\section{Discretization}

\section{SETTINGS}

\begin{tabular}{|l|l|}
\hline Description & Value \\
\hline Concentration & Quadratic \\
\hline Compute boundary fluxes & On \\
\hline
\end{tabular}




\begin{tabular}{|l|l|}
\hline Description & Value \\
\hline Apply smoothing to boundary fluxes & On \\
\hline
\end{tabular}

Transport mechanisms

\section{SETTINGS}

\begin{tabular}{|l|l|}
\hline Description & Value \\
\hline Convection & Off \\
\hline Migration in electric field & Off \\
\hline Mass transfer in porous media & Off \\
\hline
\end{tabular}

\subsubsection{Transport Properties 1}

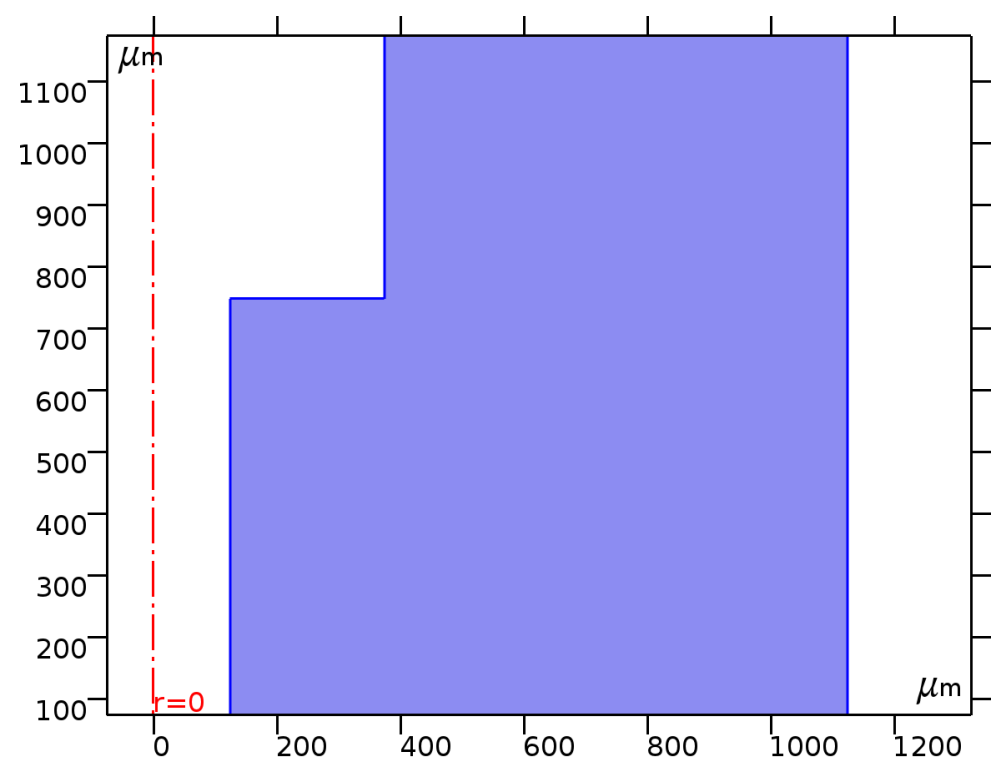

Transport Properties 1

\section{SELECTION}

\begin{tabular}{|l|l|}
\hline Geometric entity level & Domain \\
\hline Selection & Domain 2 \\
\hline
\end{tabular}

EQUATIONS

$$
\begin{aligned}
& \frac{\partial c_{i}}{\partial t}+\nabla \cdot\left(-D_{i} \nabla c_{i}\right)=R_{i} \\
& \mathbf{N}_{i}=-D_{i} \nabla c_{i}
\end{aligned}
$$

Diffusion

SETTINGS

\begin{tabular}{|l|l|}
\hline Description & Value \\
\hline Material & None \\
\hline
\end{tabular}




\begin{tabular}{|c|c|}
\hline Description & Value \\
\hline Diffusion coefficient & User defined \\
\hline Diffusion coefficient & $\{\{$ D_Fc_aq, 0,0$\},\{0$, D_Fc_aq, 0$\},\{0,0$, D_Fc_aq $\}\}$ \\
\hline Diffusion coefficient & User defined \\
\hline Diffusion coefficient & $\{\{$ D_Fc_plus_aq, 0,0$\},\{0$, D_Fc_plus_aq, 0$\},\{0,0$, D_Fc_plus_aq $\}\}$ \\
\hline Coordinate system & Global coordinate system \\
\hline Temperature & User defined \\
\hline Temperature & $293.15[\mathrm{~K}]$ \\
\hline
\end{tabular}

\section{USED PRODUCTS}

COMSOL Multiphysics

Shape functions

\begin{tabular}{|l|l|l|l|l|l|}
\hline Name & Shape function & Unit & Description & Shape frame & Selection \\
\hline Fc_aq & Lagrange (Quadratic) & $\mathrm{mol} / \mathrm{m}^{3}$ & Concentration & Material & Domain 2 \\
\hline Fc_plus_aq & Lagrange (Quadratic) & $\mathrm{mol} / \mathrm{m}^{3}$ & Concentration & Material & Domain 2 \\
\hline
\end{tabular}

\subsubsection{Axial Symmetry 1}

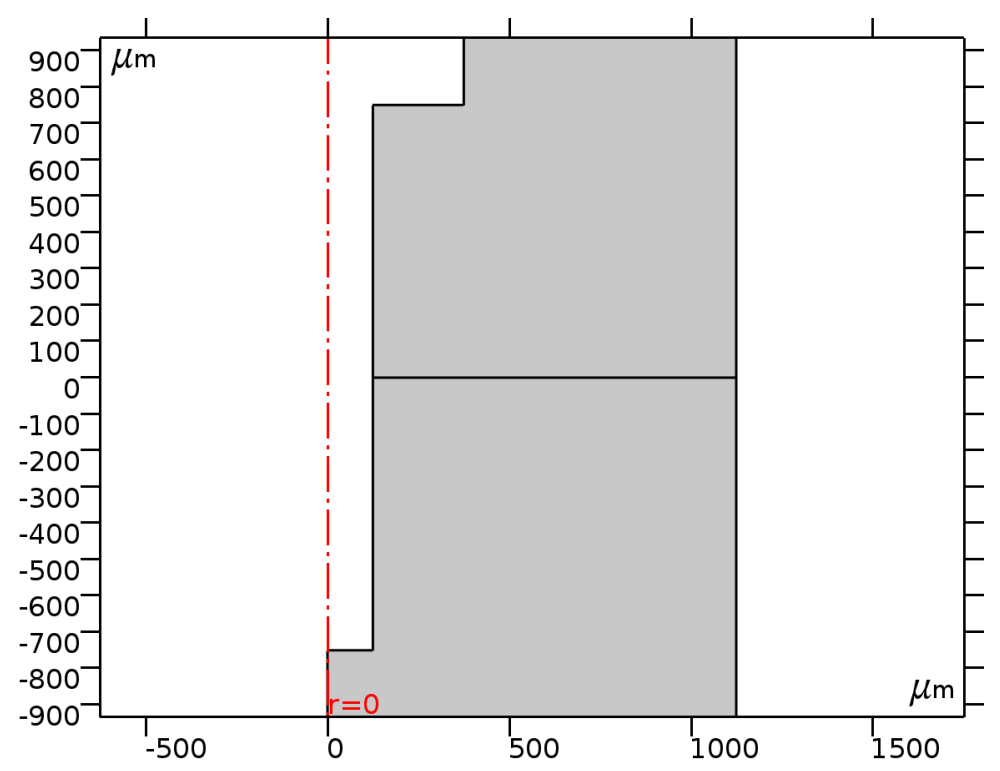

Axial Symmetry 1

\section{SELECTION}

\begin{tabular}{|l|l|}
\hline Geometric entity level & Boundary \\
\hline Selection & No boundaries
\end{tabular}

\section{USED PRODUCTS}


COMSOL Multiphysics

\subsubsection{No Flux 1}

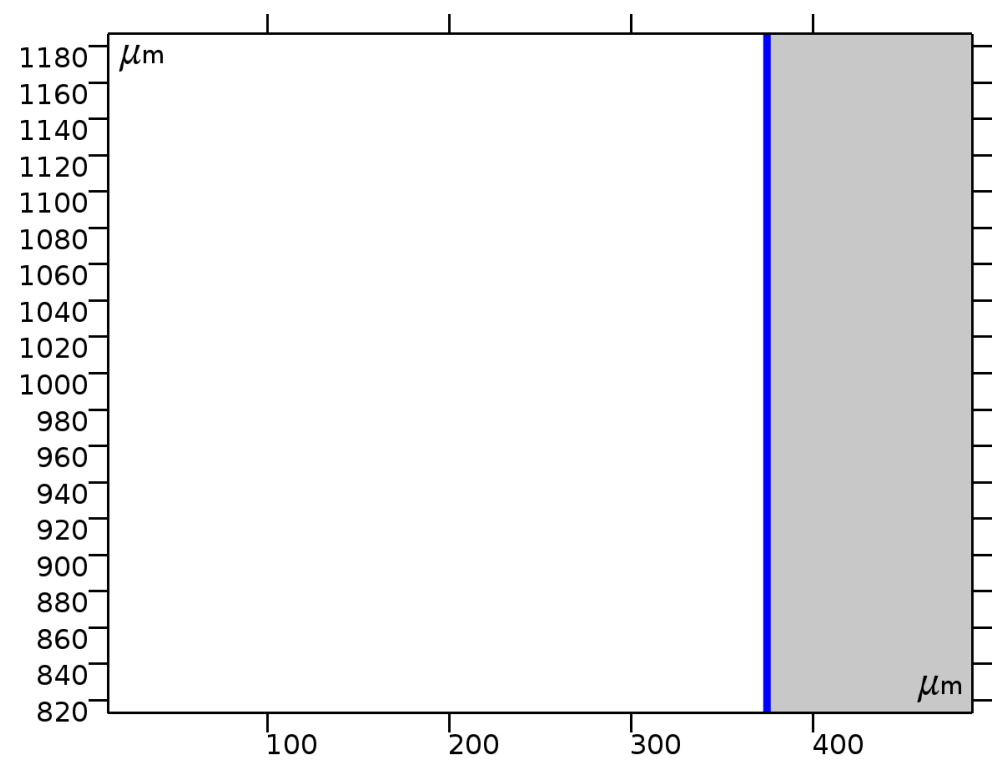

No Flux 1

SELECTION

Geometric entity level Boundary

Selection

Boundaries 7-8

EQUATIONS

$-\mathbf{n} \cdot \mathbf{N}_{i}=0$

USED PRODUCTS

COMSOL Multiphysics 


\subsubsection{Initial Values 1}

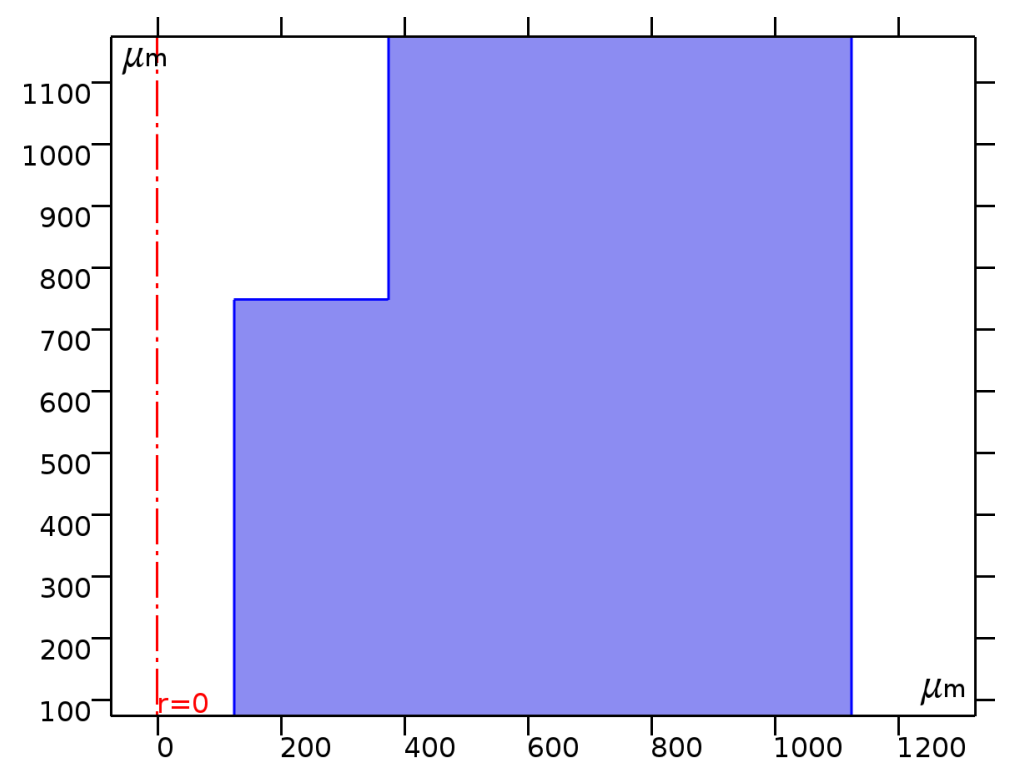

Initial Values 1

SELECTION

\begin{tabular}{|l|l|}
\hline Geometric entity level & Domain \\
\hline Selection & Domain 2
\end{tabular}

Initial values

SETTINGS

Description Value

Concentration $\{0,0\}$

USED PRODUCTS

COMSOL Multiphysics 


\subsubsection{Interfacial Fluxes}

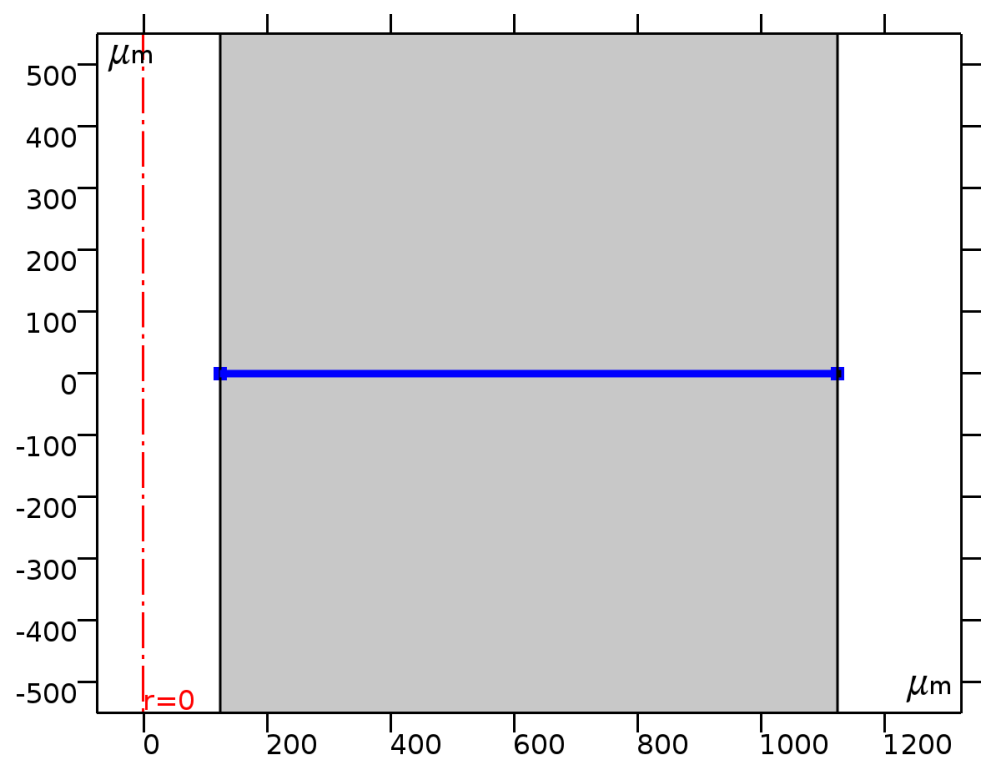

\section{Interfacial Fluxes}

\section{SELECTION}

\begin{tabular}{l|l}
\hline Geometric entity level & Boundary \\
\hline Selection & Boundary 6 \\
\hline
\end{tabular}

EQUATIONS

$-\mathbf{n} \cdot \mathbf{N}_{i}=N_{0,}$

Inward flux

SETTINGS

\begin{tabular}{|l|l|}
\hline Description & Value \\
\hline Flux type & General inward flux \\
\hline Species Fc_aq & On \\
\hline Species Fc_plus_aq & On \\
\hline & $\left\{-\left(P \_F c^{*} F c \_a q-F c \_o r g\right)^{*} k p t,-\left(P \_F c \_p l u{ }^{*} F c \_p l u s \_a q-F c \_p l u s \_o r g\right) * k p t\right\}$ \\
\hline
\end{tabular}

\section{USED PRODUCTS}

COMSOL Multiphysics

Weak expressions

\begin{tabular}{|l|l|l|l|}
\hline Weak expression & Integration order & Integration frame & Selection \\
\hline$-2^{*}\left(\mathrm{P} \_\mathrm{Fc}^{*} \mathrm{Fc} \_\mathrm{aq}-\right.$ & 4 & Material & Boundary 6 \\
\hline $\mathrm{Fc} \_$org $)^{*}{ }^{*} \mathrm{kpt}{ }^{*} \mathrm{test}\left(\mathrm{Fc} \_\mathrm{aq}\right){ }^{*}{ }^{*}{ }^{*}{ }^{*} \mathrm{r}$ & 4 & &
\end{tabular}




\begin{tabular}{|l|l|l|l|}
\hline Weak expression & Integration order & Integration frame & Selection \\
\hline $\begin{array}{l}-2^{*}\left(\mathrm{P} \_F c \_p l u{ }^{*} \mathrm{Fc} \text { _plus_aq- }\right. \\
\text { Fc_plus_org) }{ }^{*} \mathrm{kpt}{ }^{*} \text { test(Fc_plus_aq) }\end{array}$ & 4 & Material & Boundary 6 \\
\hline pi $^{*} \mathrm{r}$ & 4 & & \\
\hline
\end{tabular}

\subsubsection{Electrode Flux}

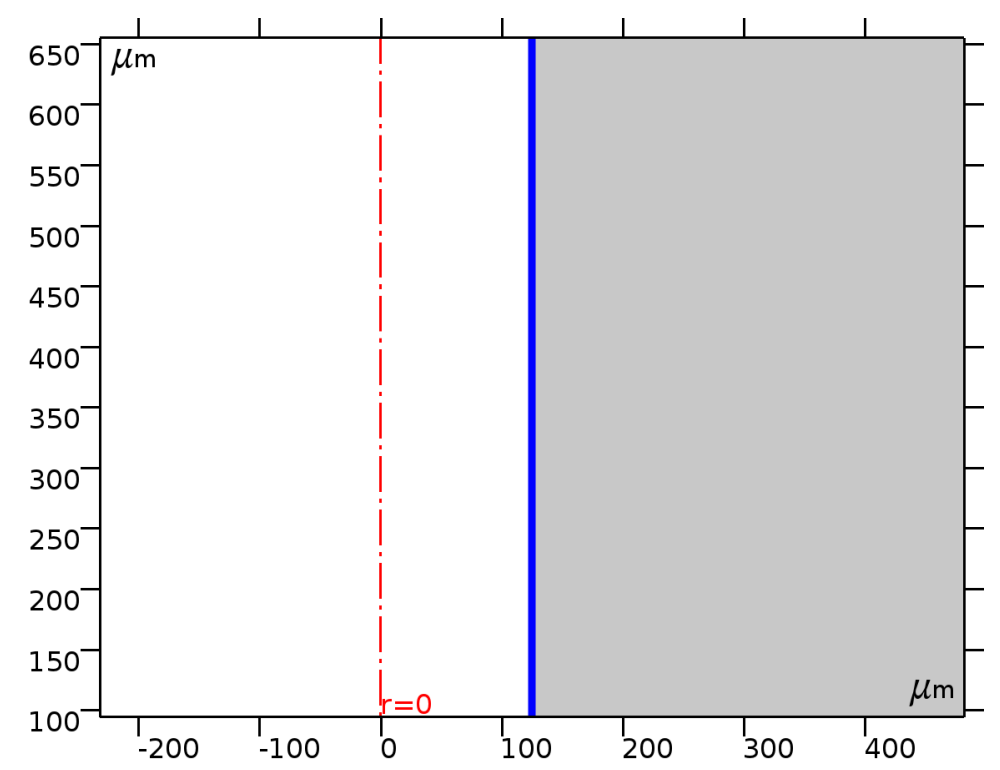

\section{Electrode Flux}

\section{SELECTION}

\begin{tabular}{|l|l|}
\hline Geometric entity level & Boundary \\
\hline Selection & Boundary 5 \\
\hline
\end{tabular}

\section{EQUATIONS}

$-\mathbf{n} \cdot \mathbf{N}_{i}=N_{0, j}$

Inward flux

SETTINGS

\begin{tabular}{|c|c|}
\hline Description & Value \\
\hline Flux type & General inward flux \\
\hline Species Fc_aq & On \\
\hline \multirow[t]{2}{*}{ Species Fc_plus_aq } & On \\
\hline & 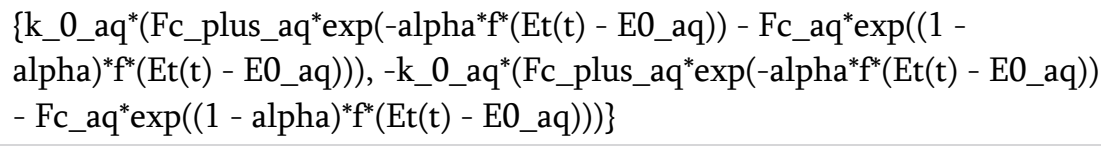 \\
\hline
\end{tabular}

\section{USED PRODUCTS}

COMSOL Multiphysics 
Weak expressions

\begin{tabular}{|c|c|c|c|}
\hline Weak expression & Integration order & Integration frame & Selection \\
\hline 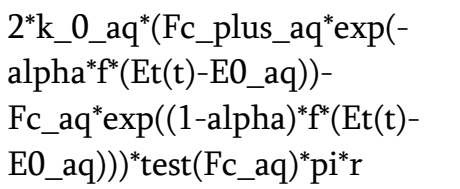 & 4 & Material & Boundary 5 \\
\hline $\begin{array}{l}-2^{*} k \_0 \_a q^{*}\left(F c \_p l u s \_a q^{*} \exp (-\right. \\
\text { alpha*f(Et(t)-E0_aq))- } \\
\text { Fc_aq*exp((1-alpha) }{ }^{*}{ }^{*}(E t(t)- \\
\text { E0_aq)))*test(Fc_plus_aq)*pi*r }\end{array}$ & 4 & Material & Boundary 5 \\
\hline
\end{tabular}

\subsubsection{Concentration 1}

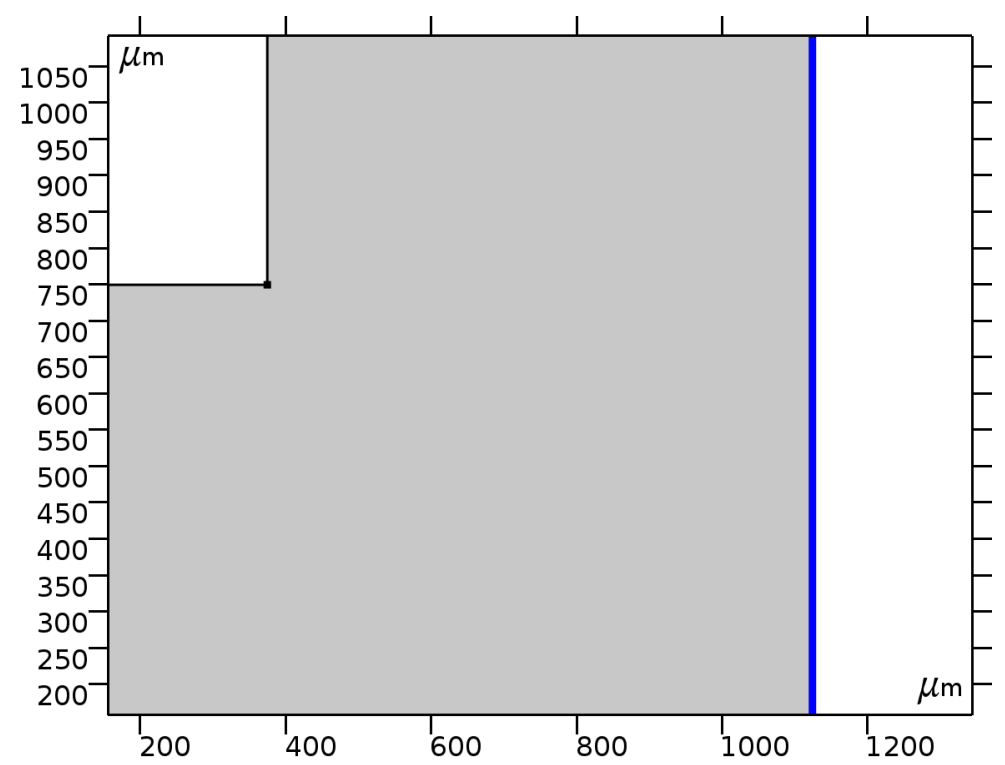

\section{Concentration 1}

SELECTION

\begin{tabular}{|l|l|}
\hline Geometric entity level & Boundary \\
\hline Selection & Boundaries 9, 11
\end{tabular}

\section{EQUATIONS}

$$
c_{i}=c_{0, j}
$$

\section{Concentration}

\section{SETTINGS}

\begin{tabular}{|l|l|}
\hline Description & Value \\
\hline Species Fc_aq & On \\
\hline Species Fc_plus_aq & On \\
\hline
\end{tabular}




\begin{tabular}{|l|l|}
\hline Description & Value \\
\hline Concentration & $\{0,0\}$ \\
\hline
\end{tabular}

\section{USED PRODUCTS}

COMSOL Multiphysics

Variables

\begin{tabular}{|l|l|l|l|l|}
\hline Name & Expression & Unit & Description & Selection \\
\hline tds3.c0_Fc_aq & 0 & $\mathrm{~mol} / \mathrm{m}^{3}$ & Concentration & Boundaries 9, 11 \\
\hline tds3.c0_Fc_plus_aq & 0 & $\mathrm{~mol} / \mathrm{m}^{3}$ & Concentration & Boundaries 9, 11 \\
\hline
\end{tabular}

Constraints

\begin{tabular}{|c|c|c|c|c|}
\hline Constraint & Constraint force & Shape function & Selection & Details \\
\hline $\begin{array}{l}- \\
\text { Fc_aq+tds3.c0_Fc_ } \\
\text { aq }\end{array}$ & $\begin{array}{l}\text { test }(- \\
\mathrm{Fc}_{-} \mathrm{aq}+\mathrm{tds} 3 . \mathrm{c} 0 \_\mathrm{Fc}_{-} \\
\text {aq) }\end{array}$ & $\begin{array}{l}\text { Lagrange } \\
\text { (Quadratic) }\end{array}$ & Boundaries 9, 11 & Elemental \\
\hline $\begin{array}{l}- \\
\text { Fc_plus_aq+tds3.c } \\
\text { 0_Fc_plus_aq }\end{array}$ & $\begin{array}{l}\text { test(- } \\
\text { Fc_plus_aq+tds3.c } \\
\text { 0_Fc_plus_aq) }\end{array}$ & $\begin{array}{l}\text { Lagrange } \\
\text { (Quadratic) }\end{array}$ & Boundaries 9, 11 & Elemental \\
\hline
\end{tabular}

\subsection{TRANSPORT OF DILUTED SPECIES (DCE PHASE)}

\section{USED PRODUCTS}

COMSOL Multiphysics

Chemical Reaction Engineering Module

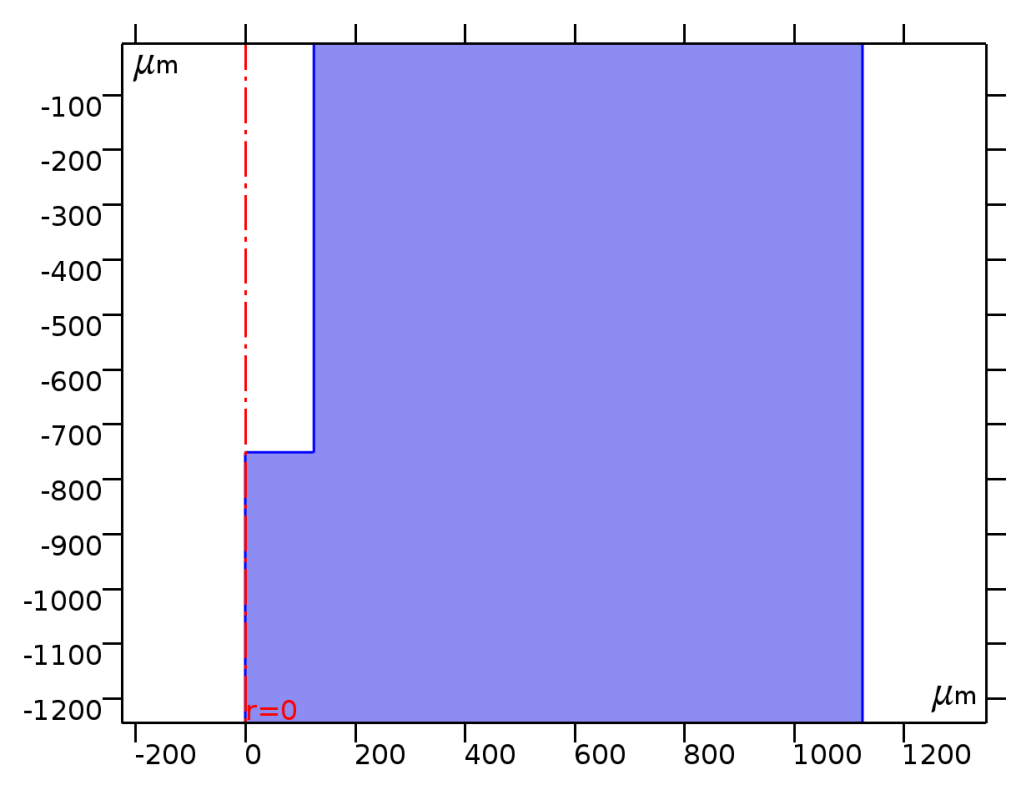

Transport of Diluted Species (DCE phase) 


\section{SELECTION}

Geometric entity level Domain

\begin{tabular}{l|l} 
Selection & Domain 1
\end{tabular}

EQUATIONS

$$
\begin{aligned}
& \frac{\partial c_{i}}{\partial \mathrm{t}}+\nabla \cdot\left(-D_{i} \nabla c_{i}\right)=R_{i} \\
& \mathbf{N}_{i}=-D_{i} \nabla c_{i}
\end{aligned}
$$

\subsubsection{Interface settings}

Discretization

\section{SETTINGS}

\begin{tabular}{|l|l|}
\hline Description & Value \\
\hline Concentration & Quadratic \\
\hline Compute boundary fluxes & On \\
\hline Apply smoothing to boundary fluxes & On \\
\hline
\end{tabular}

Transport mechanisms

\section{SETTINGS}

\begin{tabular}{|l|l|}
\hline Description & Value \\
\hline Convection & Off \\
\hline Migration in electric field & Off \\
\hline Mass transfer in porous media & Off \\
\hline
\end{tabular}




\subsubsection{Transport Properties 1}

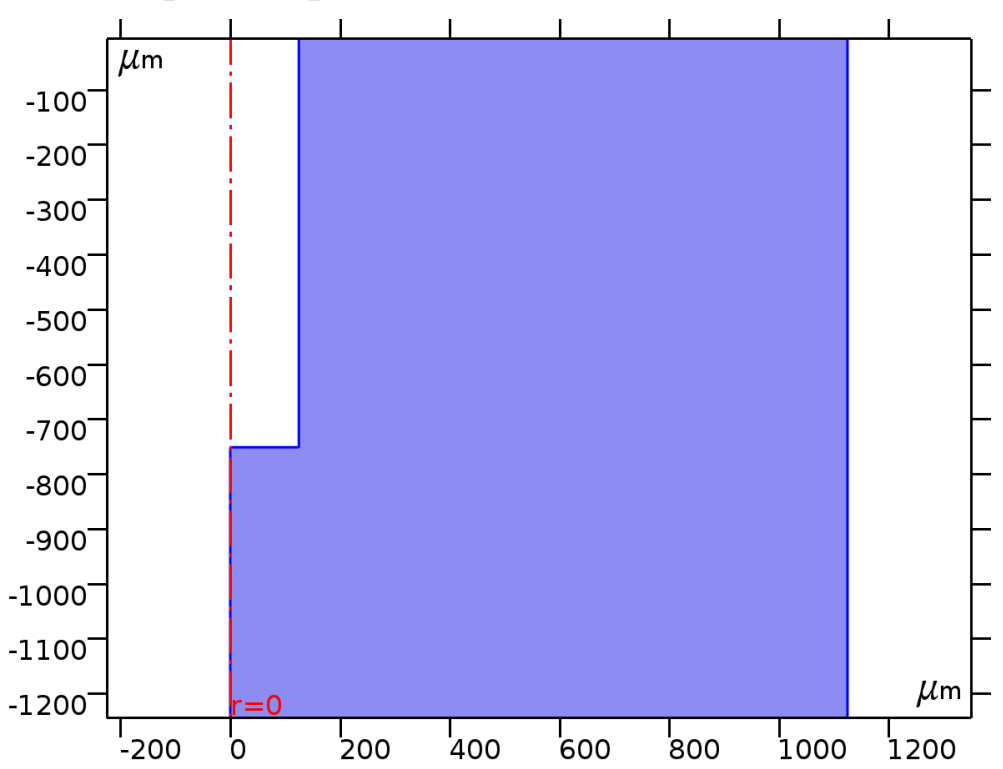

Transport Properties 1

\section{SELECTION}

\begin{tabular}{|l|l|}
\hline Geometric entity level & Domain \\
\hline Selection & Domain 1
\end{tabular}

EQUATIONS

$$
\begin{aligned}
& \frac{\partial c_{i}}{\partial t}+\nabla \cdot\left(-D_{i} \nabla c_{i}\right)=R_{i} \\
& \mathbf{N}_{i}=-D_{i} \nabla c_{i}
\end{aligned}
$$

\section{Diffusion}

\section{SETTINGS}

\begin{tabular}{|l|l|}
\hline Description & Value \\
\hline Material & None \\
\hline Diffusion coefficient & User defined \\
\hline Diffusion coefficient & $\left\{\{\right.$ D_Fc_org, 0,0\},\{0, D_Fc_org, 0 $\left.\},\left\{0,0, D \_F c \_o r g\right\}\right\}$ \\
\hline Diffusion coefficient & User defined \\
\hline Diffusion coefficient & $\{\{$ D_Fc_plus_org, 0, 0 $\},\{0$, D_Fc_plus_org, 0\}, 00, 0, D_Fc_plus_org $\}\}$ \\
\hline Coordinate system & Global coordinate system \\
\hline Temperature & User defined \\
\hline Temperature & $293.15[K]$ \\
\hline
\end{tabular}

USED PRODUCTS

COMSOL Multiphysics 
Shape functions

\begin{tabular}{|l|l|l|l|l|l|}
\hline Name & $\begin{array}{l}\text { Shape } \\
\text { function }\end{array}$ & Unit & Description & $\begin{array}{l}\text { Shape } \\
\text { frame }\end{array}$ & Selection \\
\hline Fc_org & $\begin{array}{l}\text { Lagrange } \\
\text { (Quadratic) }\end{array}$ & $\mathrm{mol} / \mathrm{m}^{3}$ & Concentration & Material & Domain 1 \\
\hline $\begin{array}{l}\text { Fc_plus } \\
\text { _org }\end{array}$ & $\begin{array}{l}\text { Lagrange } \\
\text { (Quadratic) }\end{array}$ & $\mathrm{mol} / \mathrm{m}^{3}$ & Concentration & Material & Domain 1 \\
\hline
\end{tabular}

\subsubsection{Axial Symmetry 1}

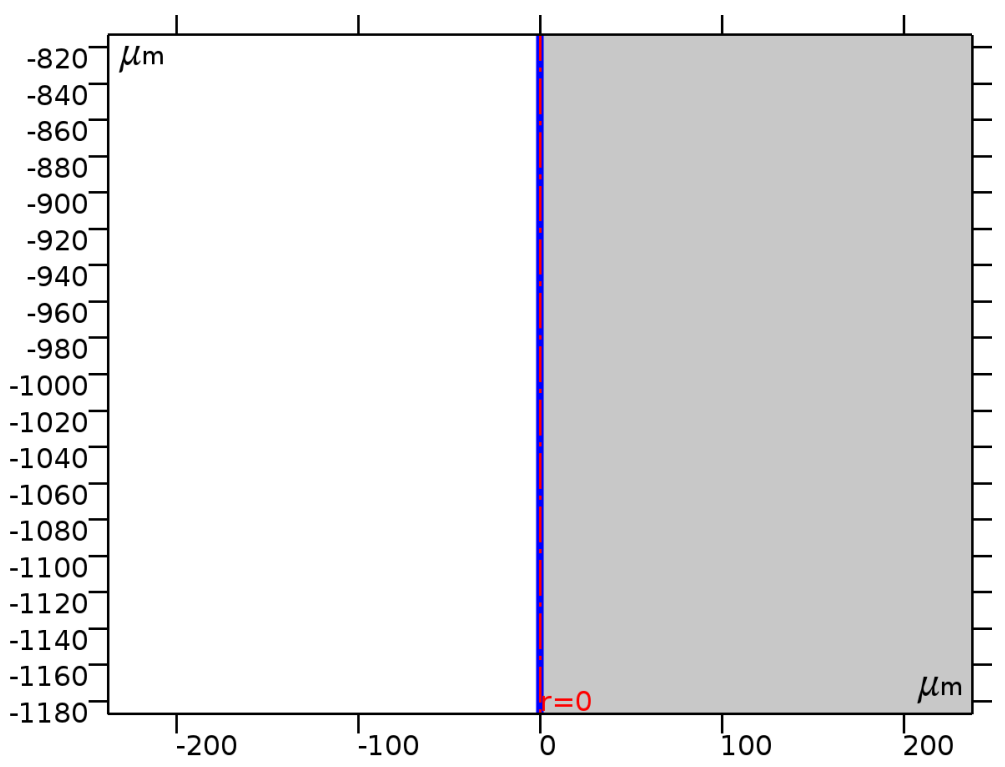

Axial Symmetry 1

\section{SELECTION}

Geometric entity level Boundary

\begin{tabular}{l|l} 
Selection & Boundary 1
\end{tabular}

\section{USED PRODUCTS}

COMSOL Multiphysics 


\subsubsection{No Flux 1}

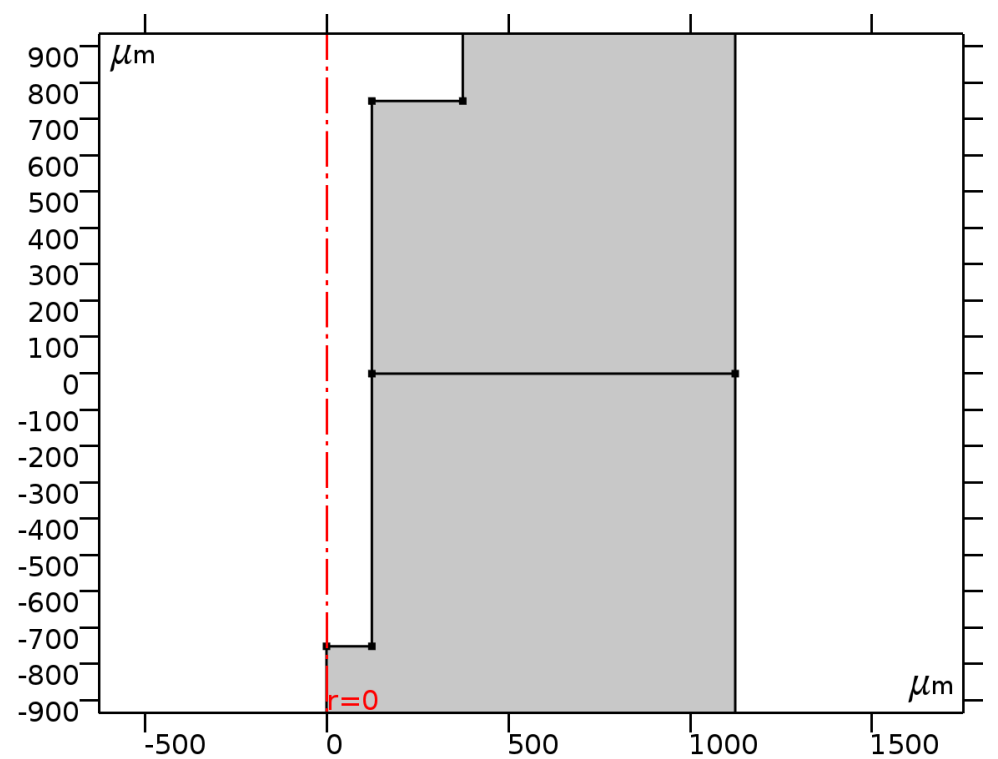

No Flux 1

SELECTION

\begin{tabular}{|l|l|}
\hline Geometric entity level & Boundary \\
\hline Selection & No boundaries
\end{tabular}

EQUATIONS

$-\mathbf{n} \cdot \mathbf{N}_{i}=0$

USED PRODUCTS

COMSOL Multiphysics 


\subsubsection{Initial Values 1}

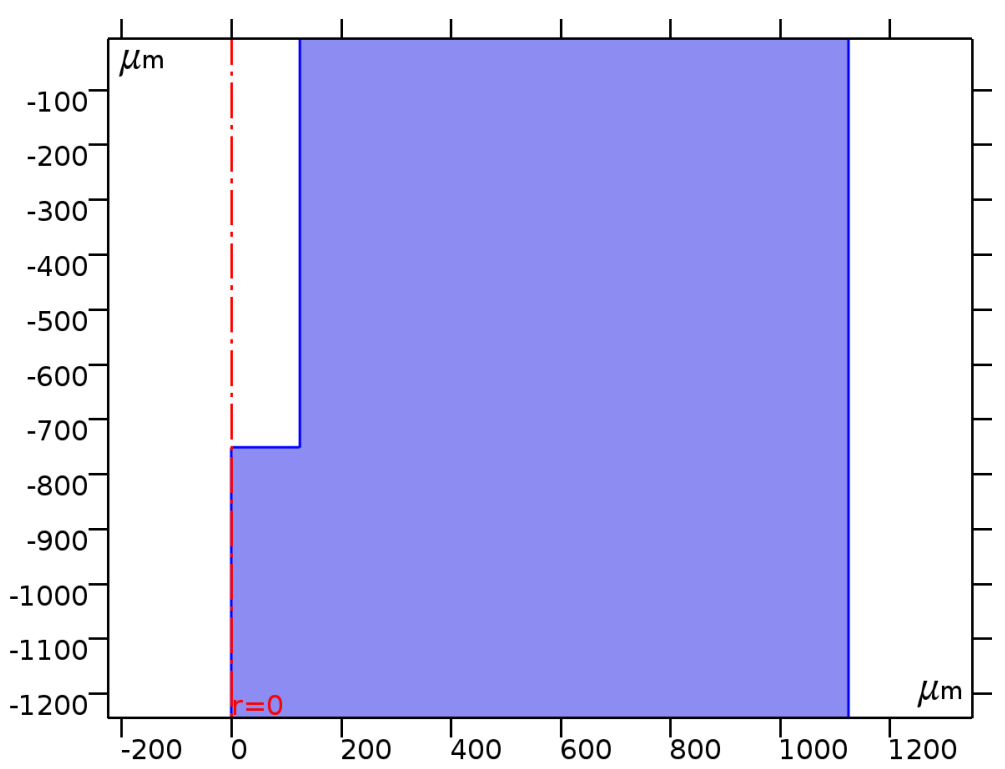

Initial Values 1

SELECTION

\begin{tabular}{|l|l|}
\hline Geometric entity level & Domain \\
\hline Selection & Domain 1
\end{tabular}

Initial values

SETTINGS

Description Value

Concentration \{c_Fc_org, 0\}

USED PRODUCTS

COMSOL Multiphysics 


\subsubsection{Interfacial Fluxes}

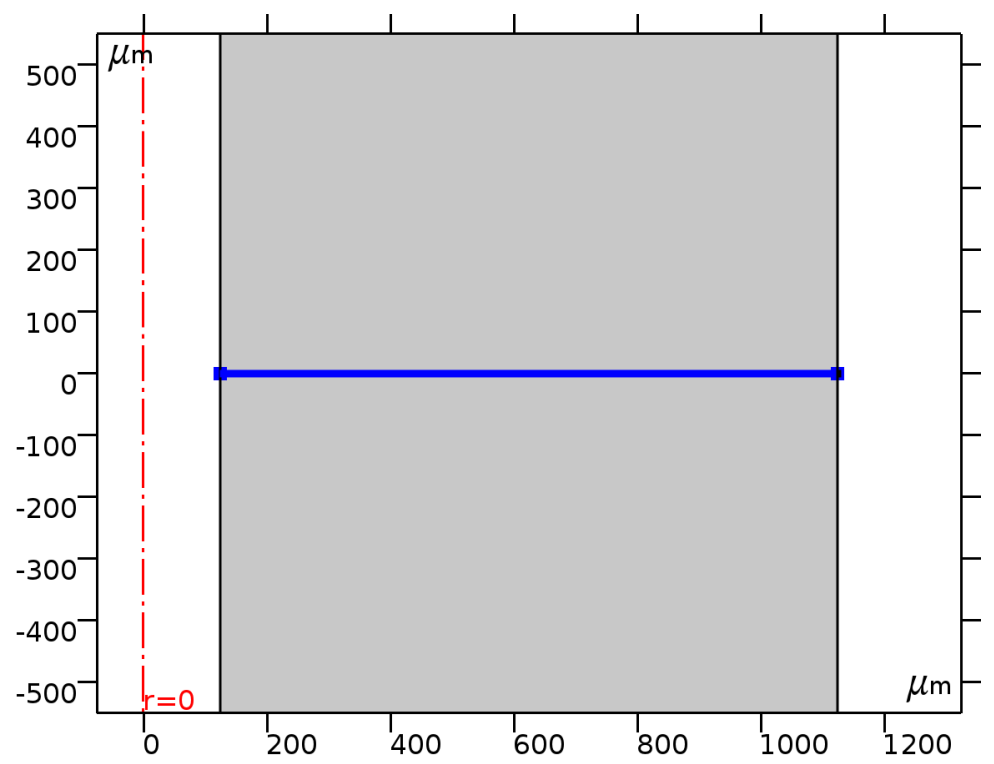

\section{Interfacial Fluxes}

\section{SELECTION}

\begin{tabular}{l|l}
\hline Geometric entity level & Boundary \\
\hline Selection & Boundary 6 \\
\hline
\end{tabular}

EQUATIONS

$-\mathbf{n} \cdot \mathbf{N}_{i}=N_{0,}$

Inward flux

\section{SETTINGS}

\begin{tabular}{|c|c|}
\hline Description & Value \\
\hline Flux type & General inward flux \\
\hline Species Fc_org & On \\
\hline \multirow[t]{2}{*}{ Species Fc_plus_org } & On \\
\hline & 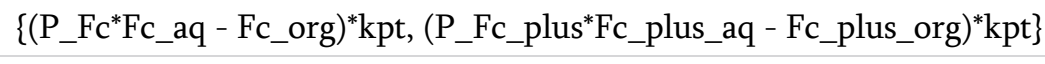 \\
\hline
\end{tabular}

\section{USED PRODUCTS}

COMSOL Multiphysics

Weak expressions

\begin{tabular}{l|l|l|l}
\hline Weak expression & Integration order & Integration frame & Selection \\
\hline $\begin{array}{l}2^{*}\left(\mathrm{P} \_F c^{*} \mathrm{FC} \_\mathrm{aq}-\right. \\
\left.\mathrm{Fc} \_ \text {org }\right)^{*} \mathrm{kpt}{ }^{*} \mathrm{test}\left(\mathrm{FC} \_ \text {org }\right)^{*} \mathrm{pi}^{*} \mathrm{r}\end{array}$ & 4 & Material & Boundary 6
\end{tabular}




\begin{tabular}{|l|l|l|l|}
\hline Weak expression & Integration order & Integration frame & Selection \\
\hline $\begin{array}{l}\text { 2*(P_Fc_plus*Fc_plus_aq- } \\
\text { Fc_plus_org) }{ }^{*} \text { kpt*test(Fc_plus_org) } \\
{ }^{*} \text { pi }^{*} \text { r }\end{array}$ & 4 & Material & Boundary 6 \\
\hline
\end{tabular}

\subsubsection{Electrode Flux}

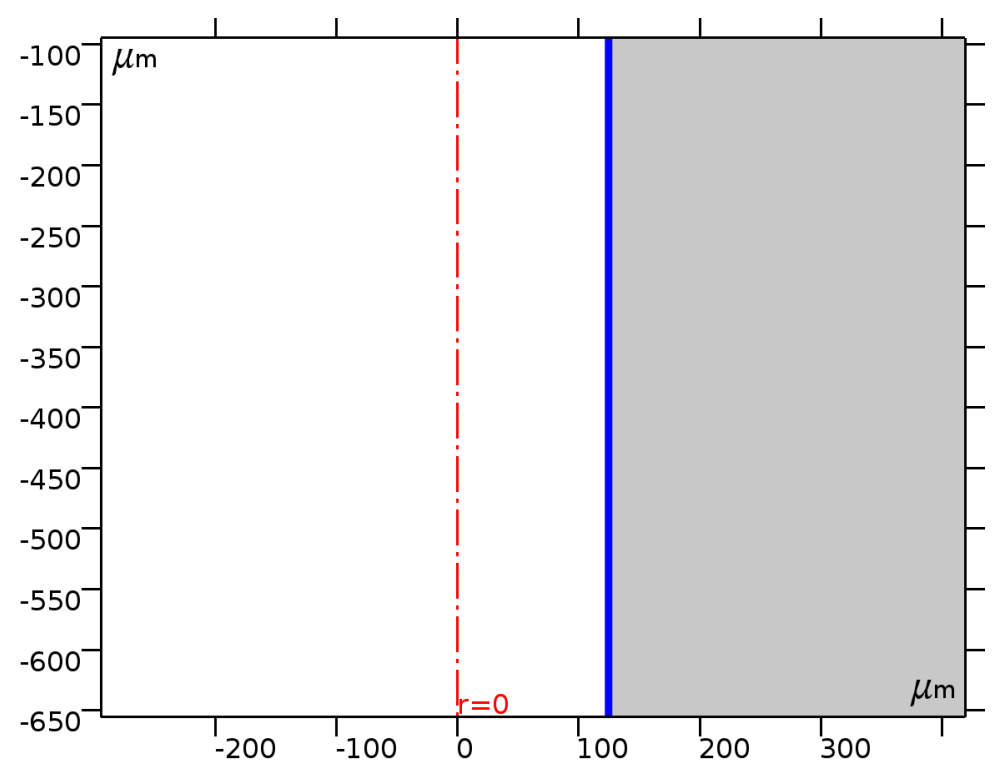

\section{Electrode Flux}

\section{SELECTION}

Geometric entity level Boundary

\begin{tabular}{l|l} 
Selection & Boundaries 3-4
\end{tabular}

\section{EQUATIONS}

$-\mathbf{n} \cdot \mathbf{N}_{i}=N_{0,}$

Inward flux

SETTINGS

\begin{tabular}{|c|c|}
\hline Description & Value \\
\hline Flux type & General inward flux \\
\hline Species Fc_org & On \\
\hline \multirow{2}{*}{ Species Fc_plus_org } & On \\
\hline & 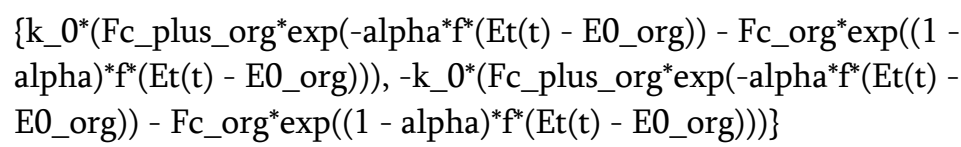 \\
\hline
\end{tabular}

\section{USED PRODUCTS}

COMSOL Multiphysics 
Weak expressions

\begin{tabular}{|c|c|c|c|}
\hline Weak expression & Integration order & Integration frame & Selection \\
\hline 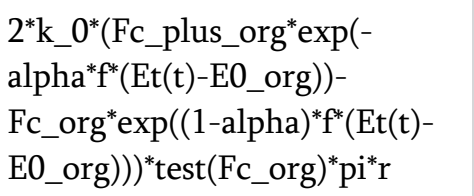 & 4 & Material & Boundaries 3-4 \\
\hline 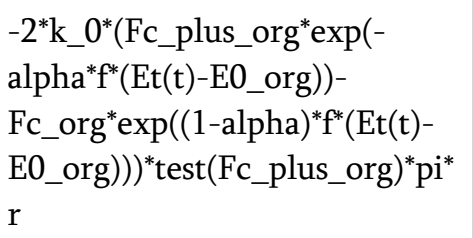 & 4 & Material & Boundaries 3-4 \\
\hline
\end{tabular}

\subsubsection{Concentration 1}

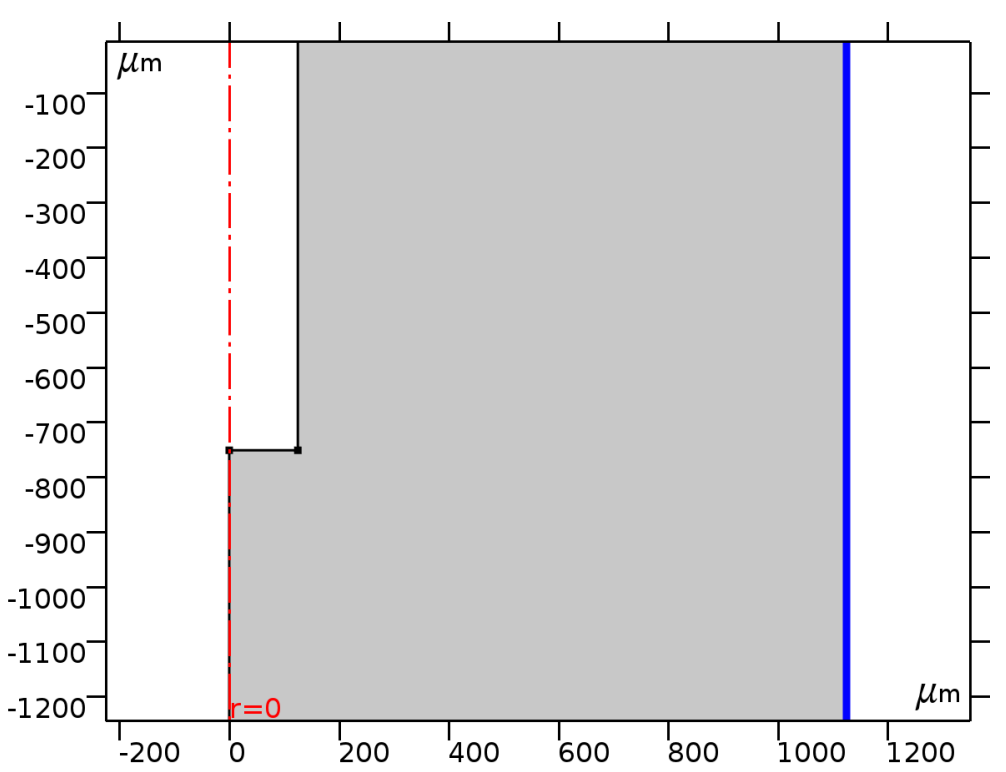

Concentration 1

SELECTION

Geometric entity level Boundary

Selection $\quad$ Boundaries 2, 10

EQUATIONS

$c_{i}=c_{0, j}$

Concentration

SETTINGS

\begin{tabular}{|l|l|}
\hline Description & Value \\
\hline Species Fc_org & On \\
\hline
\end{tabular}




\begin{tabular}{|l|l|}
\hline Description & Value \\
\hline Species Fc_plus_org & On \\
\hline Concentration & $\{$ c_Fc_org, 0 \\
\hline
\end{tabular}

\section{USED PRODUCTS}

COMSOL Multiphysics

Variables

\begin{tabular}{|l|l|l|l|l|}
\hline Name & Expression & Unit & Description & Selection \\
\hline tds4.c0_Fc_org & c_Fc_org & $\mathrm{mol} / \mathrm{m}^{3}$ & Concentration & Boundaries 2, 10 \\
\hline tds4.c0_Fc_plus_org & 0 & $\mathrm{~mol} / \mathrm{m}^{3}$ & Concentration & Boundaries 2, 10 \\
\hline
\end{tabular}

Constraints

\begin{tabular}{|c|c|c|c|c|}
\hline Constraint & Constraint force & Shape function & Selection & Details \\
\hline $\begin{array}{l}- \\
\text { Fc_org+tds4.c0_Fc } \\
\text { _org }\end{array}$ & $\begin{array}{l}\text { test(- } \\
\text { Fc_org+tds4.c0_Fc } \\
\text { _org) }\end{array}$ & $\begin{array}{l}\text { Lagrange } \\
\text { (Quadratic) }\end{array}$ & Boundaries 2, 10 & Elemental \\
\hline $\begin{array}{l}- \\
\text { Fc_plus_org+tds4.c } \\
\text { 0_Fc_plus_org }\end{array}$ & $\begin{array}{l}\text { test(- } \\
\text { Fc_plus_org+tds4.c } \\
\text { 0_Fc_plus_org) }\end{array}$ & $\begin{array}{l}\text { Lagrange } \\
\text { (Quadratic) }\end{array}$ & Boundaries 2, 10 & Elemental \\
\hline
\end{tabular}

\subsection{MESH}

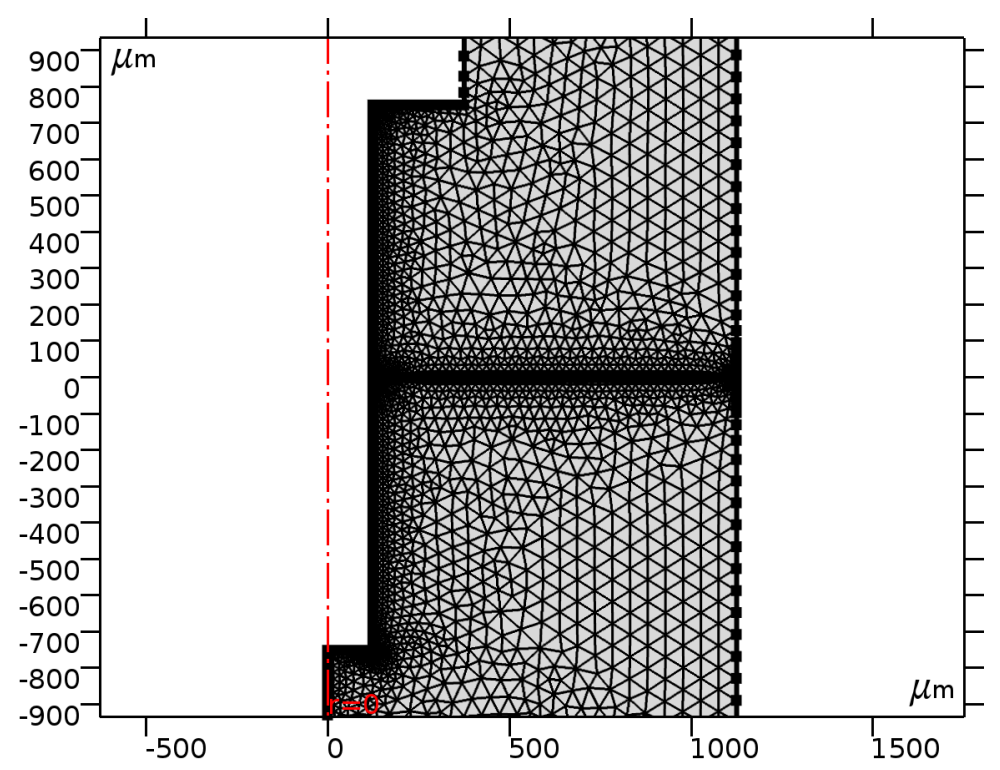

Diffusion_only

\subsubsection{Size (size)}

\section{SETTINGS}




\begin{tabular}{|l|l|}
\hline Description & Value \\
\hline Maximum element size & 60 \\
\hline Minimum element size & 0.075 \\
\hline Curvature factor & 0.25 \\
\hline Maximum element growth rate & 1.25 \\
\hline Predefined size & Extra fine \\
\hline Custom element size & Custom \\
\hline
\end{tabular}

\subsubsection{Size 2 (size2)}

\section{SELECTION}

\begin{tabular}{|l|l|}
\hline Geometric entity level & Point \\
\hline Selection & Points 3-5, 9 \\
\hline
\end{tabular}

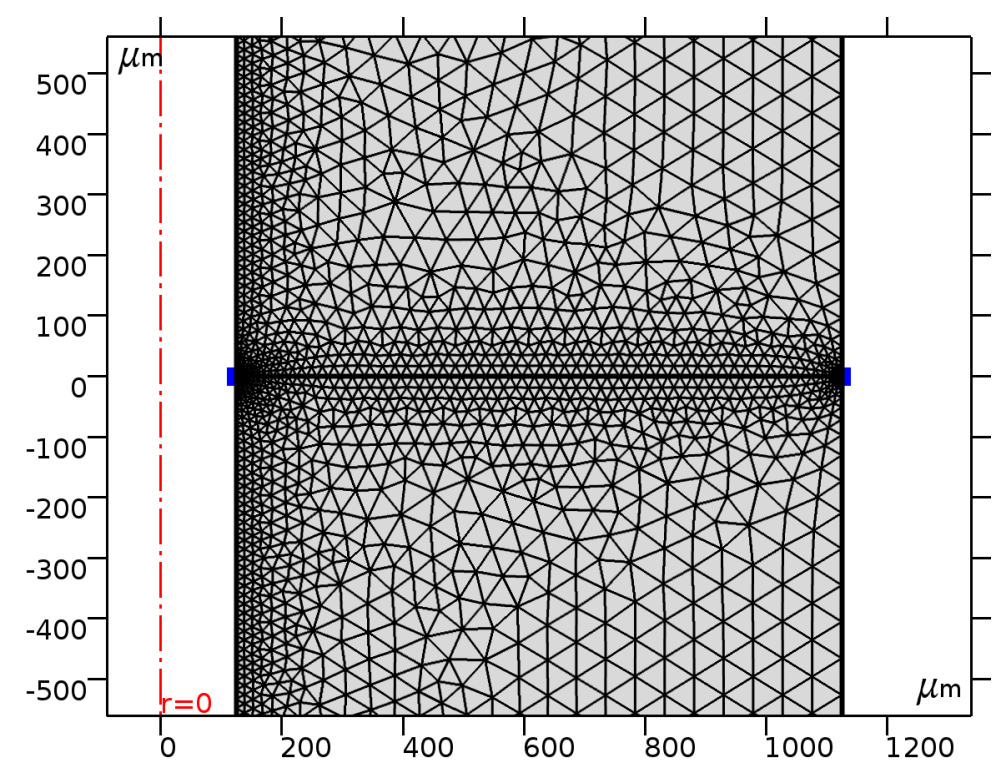

Size 2

\section{SETTINGS}

\begin{tabular}{|l|l|}
\hline Description & Value \\
\hline Maximum element size & 5 \\
\hline Minimum element size & 0.48 \\
\hline Minimum element size & Off \\
\hline Curvature factor & 0.3 \\
\hline Curvature factor & Off \\
\hline Resolution of narrow regions & Off \\
\hline Maximum element growth rate & 1.3 \\
\hline Maximum element growth rate & Off \\
\hline
\end{tabular}




\begin{tabular}{|l|l|}
\hline Description & Value \\
\hline Custom element size & Custom \\
\hline
\end{tabular}

\subsubsection{Size 1 (size1)}

\section{SELECTION}

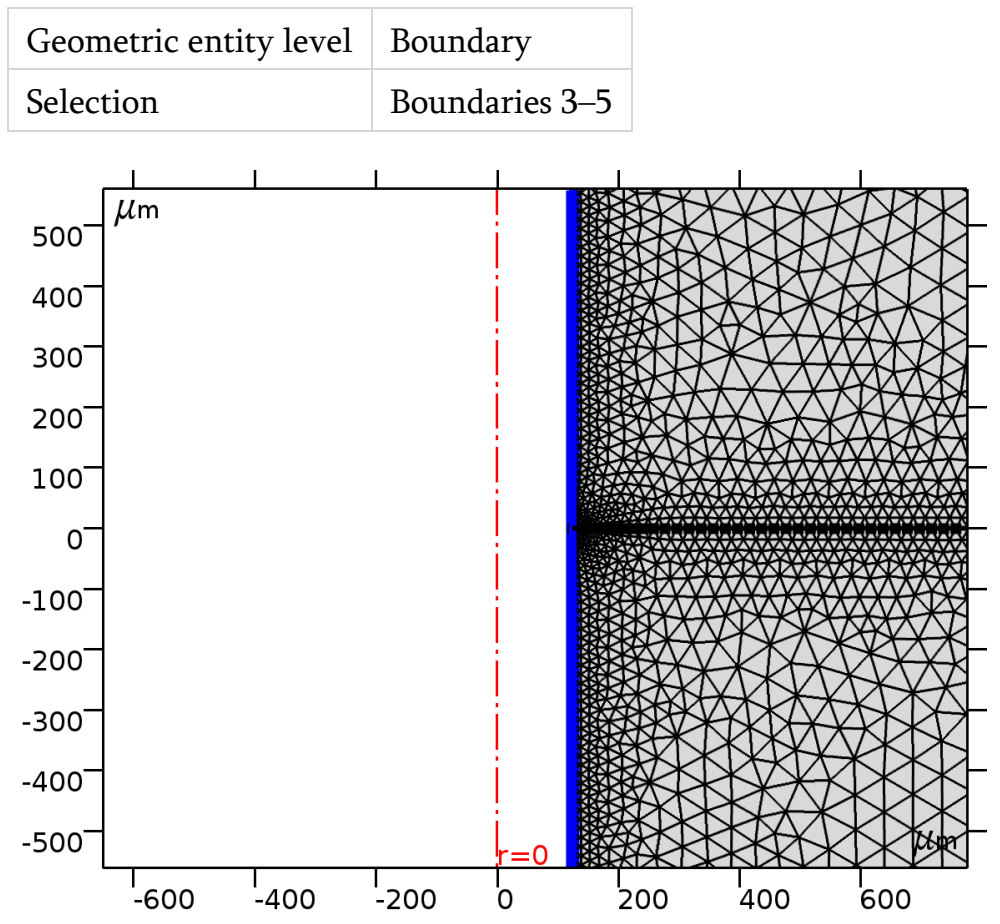

Size 1

\section{SETTINGS}

\begin{tabular}{|l|l|}
\hline Description & Value \\
\hline Maximum element size & 15 \\
\hline Minimum element size & 0.02 \\
\hline Minimum element size & Off \\
\hline Curvature factor & 0.2 \\
\hline Curvature factor & Off \\
\hline Resolution of narrow regions & Off \\
\hline Predefined size & Extremely fine \\
\hline Custom element size & Custom \\
\hline
\end{tabular}

\subsubsection{Size 3 (size3)}

\section{SELECTION}

\begin{tabular}{|l|l|}
\hline Geometric entity level & Boundary \\
\hline Selection & Boundary 6 \\
\hline
\end{tabular}




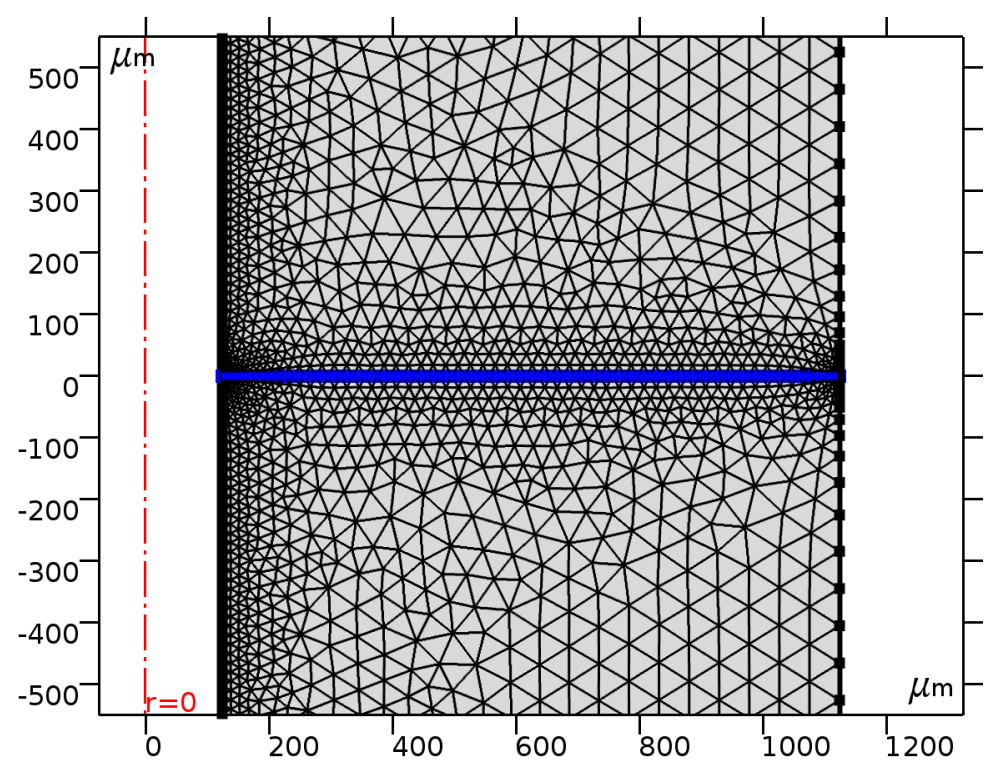

Size 3

SETTINGS
\begin{tabular}{|l|l|}
\hline Description & Value \\
\hline Maximum element size & 20 \\
\hline Minimum element size & 0.187 \\
\hline Minimum element size & Off \\
\hline Curvature factor & 0.25 \\
\hline Curvature factor & Off \\
\hline Resolution of narrow regions & Off \\
\hline Maximum element growth rate & 1.2 \\
\hline Maximum element growth rate & Off \\
\hline Predefined size & Extra fine \\
\hline Custom element size & Custom \\
\hline
\end{tabular}

\subsubsection{Free Triangular 1 (ftri1)}

\section{SELECTION}

\begin{tabular}{|l|l|}
\hline Geometric entity level & Domain \\
\hline Selection & Remaining \\
\hline
\end{tabular}




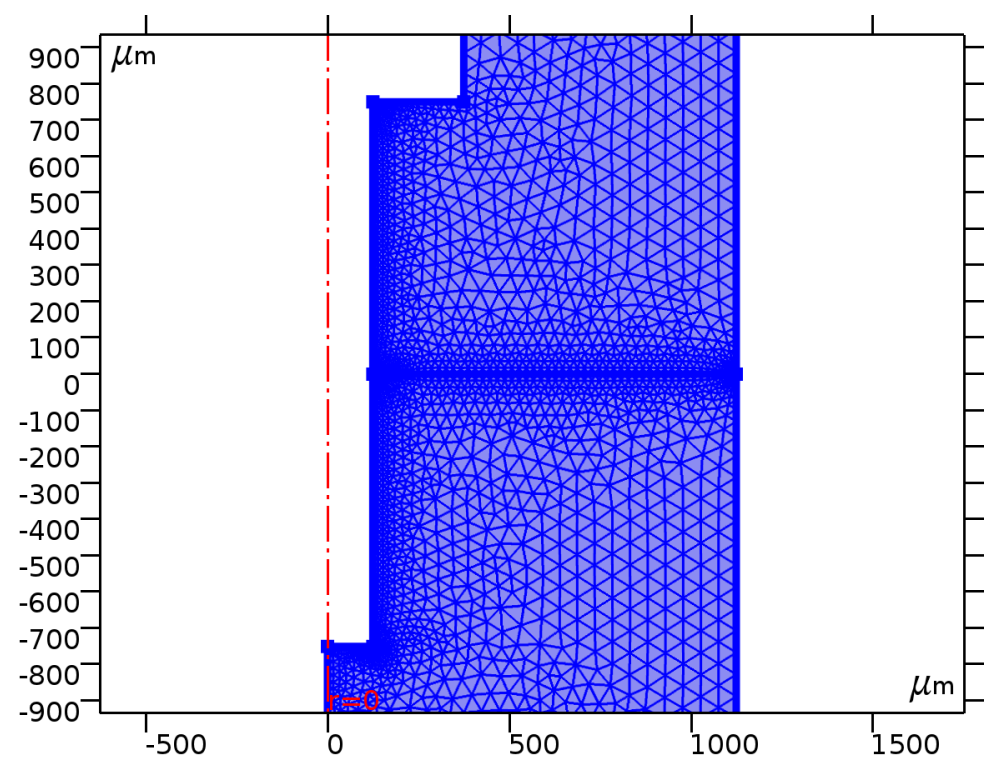

Free Triangular 1 


\section{STUDY 1}

COMPUTATION INFORMATION

\begin{tabular}{|l|l|}
\hline Computation time & $5 \min 32 \mathrm{~s}$ \\
\hline CPU & Intel64 Family 6 Model 60 Stepping 3, 4 cores \\
\hline Operating system & Windows 10 \\
\hline
\end{tabular}

\subsection{STATIONARY}

PHYSICS AND VARIABLES SELECTION

\begin{tabular}{|c|c|}
\hline Physics interface & \\
\hline Transport of Diluted & pecies (H \\
\hline Transport of Diluted & pecies (1 \\
\hline MESH SELECTION & \\
\hline Geometry & Mesh \\
\hline Geometry 1 (geom 1 ) & mesh1 \\
\hline
\end{tabular}

\subsection{TIME DEPENDENT}

\begin{tabular}{|l|l|}
\hline Times & Unit \\
\hline range $\left(0,10[\mathrm{mV}] / \mathrm{v}, \mathrm{ts}^{*} 4\right)$ & $\mathrm{s}$ \\
\hline
\end{tabular}

VALUES OF DEPENDENT VARIABLES

\begin{tabular}{|l|l|}
\hline Description & Value \\
\hline Settings & User controlled \\
\hline Method & Solution \\
\hline
\end{tabular}

PHYSICS AND VARIABLES SELECTION

\begin{tabular}{|l|l|}
\hline Physics interface & Discretiz \\
\hline Transport of Diluted Species (H2O phase) (tds3) & physics \\
\hline Transport of Diluted Species (DCE phase) (tds4) & physics \\
\hline
\end{tabular}

MESH SELECTION

\begin{tabular}{|l|l|}
\hline Geometry & Mesh \\
\hline Geometry 1 (geom1) & mesh1
\end{tabular}




\subsection{SOLVER CONFIGURATIONS}

\subsubsection{Solution 1}

Compile Equations: Stationary (st1)

STUDY AND STEP

\begin{tabular}{|l|l|}
\hline Description & Value \\
\hline Use study & Diffusion only \\
\hline Use study step & Stationary \\
\hline
\end{tabular}

Dependent Variables 1 (v1)

GENERAL

\begin{tabular}{|l|l|}
\hline Description & Value \\
\hline Defined by study step & $\underline{\text { Stationary }}$ \\
\hline
\end{tabular}

SETTINGS

\begin{tabular}{|l|l|}
\hline Constant name & Initial value source \\
\hline timestep & $0.0016[\mathrm{~s}]$ \\
\hline
\end{tabular}

Concentration (comp1.Fc_aq) (comp1_Fc_aq)

GENERAL

\begin{tabular}{|l|l|}
\hline Description & Value \\
\hline Field components & comp1.Fc_aq \\
\hline
\end{tabular}

Concentration (comp1.Fc_org) (comp1_Fc_org)

GENERAL

\begin{tabular}{|l|l|}
\hline Description & Value \\
\hline Field components & comp1.Fc_org \\
\hline
\end{tabular}

Concentration (comp1.Fc_plus_aq) (comp1_Fc_plus_aq)

GENERAL

\begin{tabular}{|l|l|}
\hline Description & Value \\
\hline Field components & comp1.Fc_plus_aq \\
\hline
\end{tabular}

Concentration (comp1.Fc_plus_org) (comp1_Fc_plus_org)

GENERAL

\begin{tabular}{|l|l|}
\hline Description & Value \\
\hline Field components & comp1.Fc_plus_org \\
\hline
\end{tabular}


Stationary Solver 1 (s1)

GENERAL

\begin{tabular}{|l|l|} 
Description & Value \\
\hline Defined by study step & $\underline{\text { Stationary }}$ \\
\hline
\end{tabular}

RESULTS WHILE SOLVING

\section{Description Value}

\begin{tabular}{l|l} 
Plot & On
\end{tabular}

Fully Coupled 1 (fc1)

GENERAL

\begin{tabular}{|l|l|}
\hline Description & Value \\
\hline Linear solver & $\underline{\text { Direct } 1}$ \\
\hline
\end{tabular}

METHOD AND TERMINATION

\begin{tabular}{|l|l|}
\hline Description & Value \\
\hline Initial damping factor & 0.01 \\
\hline Minimum damping factor & $1.0 \mathrm{E}-6$ \\
\hline Maximum number of iterations & 50
\end{tabular}

Direct 1 (d1)

GENERAL

\begin{tabular}{|l|l|} 
Description & Value \\
\hline Solver & PARDISO
\end{tabular}

Solution Store 1 (su1)

GENERAL

\section{Description Value}

Solution $\quad$ Solution Store 1

Compile Equations: Time Dependent (st2)

STUDY AND STEP

\begin{tabular}{|l|l|}
\hline Description & Value \\
\hline Use study & $\underline{\text { Diffusion only }}$ \\
\hline Use study step & $\underline{\text { Time Dependent }}$ \\
\hline
\end{tabular}

Dependent Variables 2 (v2)

GENERAL 


\begin{tabular}{|l|l|}
\hline Description & Value \\
\hline Defined by study step & Time Dependent \\
\hline
\end{tabular}

INITIAL VALUES OF VARIABLES SOLVED FOR

\begin{tabular}{|l|l|}
\hline Description & Value \\
\hline Method & Solution \\
\hline Solution & Solution 1 \\
\hline
\end{tabular}

VALUES OF VARIABLES NOT SOLVED FOR

\begin{tabular}{|l|l|} 
Description & Value \\
\hline Method & Solution \\
\hline
\end{tabular}

\section{SETTINGS}

\begin{tabular}{|l|l|}
\hline Constant name & Initial value source \\
\hline $\mathrm{t}$ & $\operatorname{range}\left(0,10[\mathrm{mV}] / \mathrm{v}, \mathrm{ts}^{*} 4\right)$ \\
\hline timestep & $0.0016[\mathrm{~s}]$ \\
\hline
\end{tabular}

Concentration (comp1.Fc_aq) (comp1_Fc_aq)

\section{GENERAL}

\begin{tabular}{|l|l|}
\hline Description & Value \\
\hline Field components & comp1.Fc_aq \\
\hline
\end{tabular}

Concentration (comp1.Fc_org) (comp1_Fc_org)

\section{GENERAL}

\begin{tabular}{|l|l|}
\hline Description & Value \\
\hline Field components & comp1.Fc_org \\
\hline
\end{tabular}

Concentration (comp1.Fc_plus_aq) (comp1_Fc_plus_aq)

GENERAL

\begin{tabular}{|l|l|}
\hline Description & Value \\
\hline Field components & comp1.Fc_plus_aq \\
\hline
\end{tabular}

Concentration (comp1.Fc_plus_org) (comp1_Fc_plus_org)

GENERAL

\begin{tabular}{|l|l|}
\hline Description & Value \\
\hline Field components & comp1.Fc_plus_org \\
\hline
\end{tabular}

Time-Dependent Solver 1 ( $t 1)$

GENERAL 


\begin{tabular}{|c|c|}
\hline Description & Value \\
\hline Times & $\begin{array}{l}\{0,0.1,0.2,0.3,0.4,0.5,0.6,0.7,0.8,0.9,1,1.1,1.2,1.3,1.4,1.5,1.6,1.7,1.8,1.9,2 \text {, } \\
2.1,2.2,2.3,2.4,2.5,2.6,2.7,2.8,2.9,3,3.1,3.2,3.3,3.4,3.5,3.6,3.7,3.8,3.9,4, \\
4.1,4.2,4.3,4.4,4.5,4.6,4.7,4.8,4.9,5,5.1,5.2,5.3,5.4,5.5,5.6,5.7,5.8,5.9,6, \\
6.1,6.2,6.3,6.4,6.5,6.6,6.7,6.8,6.9,7,7.1,7.2,7.3,7.4,7.5,7.6,7.7,7.8,7.9,8, \\
8.1,8.2,8.3,8.4,8.5,8.6,8.7,8.8,8.9,9,9.1,9.2,9.3,9.4,9.5,9.6,9.7,9.8,9.9,10, \\
10.1,10.2,10.3,10.4,10.5,10.6,10.7,10.8,10.9,11,11.1,11.2,11.3,11.4,11.5, \\
11.6,11.7,11.8,11.9,12,12.1,12.2,12.3,12.4,12.5,12.6,12.7,12.8,12.9,13,13.1, \\
13.2,13.3,13.4,13.5,13.6,13.7,13.8,13.9,14,14.1,14.2,14.3,14.4,14.5,14.6, \\
14.7,14.8,14.9,15,15.1,15.2,15.3,15.4,15.5,15.6,15.7,15.8,15.9,16,16.1,16.2, \\
16.3,16.4,16.5,16.6,16.7,16.8,16.9,17,17.1,17.2,17.3,17.4,17.5,17.6,17.7, \\
17.8,17.9,18,18.1,18.2,18.3,18.4,18.5,18.6,18.7,18.8,18.9,19,19.1,19.2,19.3, \\
19.4,19.5,19.6,19.7,19.8,19.9,20,20.1,20.2,20.3,20.4,20.5,20.6,20.7,20.8, \\
20.9,21,21.1,21.2,21.3,21.4,21.5,21.6,21.7,21.8,21.9,22,22.1,22.2,22.3,22.4, \\
22.5,22.6,22.7,22.8,22.9,23,23.1,23.2,23.3,23.4,23.5,23.6,23.7,23.8,23.9,24, \\
24.1,24.2,24.3,24.4,24.5,24.6,24.7,24.8,24.9,25,25.1,25.2,25.3,25.4,25.5, \\
25.6,25.7,25.8,25.9,26,26.1,26.2,26.3,26.4,26.5,26.6,26.7,26.8,26.9,27,27.1, \\
27.2,27.3,27.4,27.5,27.6,27.7,27.8,27.9,28,28.1,28.2,28.3,28.4,28.5,28.6, \\
28.7,28.8,28.9,29,29.1,29.2,29.3,29.4,29.5,29.6,29.7,29.8,29.9,30,30.1,30.2, \\
30.3,30.4,30.5,30.6,30.7,30.8,30.9,31,31.1,31.2,31.3,31.4,31.5,31.6,31.7, \\
31.8,31.9,32\}\end{array}$ \\
\hline
\end{tabular}

TIME STEPPING

\begin{tabular}{|l|l|}
\hline Description & Value \\
\hline Steps taken by solver & Intermediate \\
\hline Maximum BDF order & 2 \\
\hline
\end{tabular}

\section{RESULTS WHILE SOLVING}

\begin{tabular}{|l|l|}
\hline Description & Value \\
\hline Plot & On \\
\hline
\end{tabular}

Fully Coupled 1 (fc1)

GENERAL
\begin{tabular}{|l|l|l|}
\hline Description & Value & \\
\hline Linear solver & $\underline{\text { Direct } 1}$ \\
\hline
\end{tabular}
METHOD AND TERMINATION
\begin{tabular}{|l|l|}
\hline Description & Value \\
\hline Damping factor & 0.9 \\
\hline Jacobian update & Once per time step \\
\hline Maximum number of iterations & 8 \\
\hline Stabilization and acceleration & Anderson acceleration \\
\hline Dimension of iteration space & 5 \\
\hline
\end{tabular}


Direct 1 (d1)

GENERAL

Description Value

\begin{tabular}{l|l} 
Solver & PARDISO
\end{tabular} 